\title{
Water masers in NGC7538 region
}

\section{Osamu Kameya ${ }^{1}$}

National Astronomical Observatory of Japan

2-12 Hoshigaoka-cho, Mizusawa, Oshu, Iwate, Japan

E-mail: kameya@miz.nao.ac.jp

We observed $\mathrm{H}_{2} \mathrm{O}$ masers towards NGC7538 molecular-cloud core using VERA (VLBI Experiment of Radio Astrometry). This region is in the Perseus arm at a distance of about 2.7 kpc and is famous for its multiple, massive star formation. There are three areas there, N(IRS13), E(IRS9), and S(IRS11), each having a strong IR source(s), ultra-compact HII region(s), bipolar outflow, high-density core, and $\mathrm{OH} / \mathrm{H}_{2} \mathrm{O} / \mathrm{CH}_{3} \mathrm{OH}$ masers. We made differential VLBI observations towards the NGC7538 $\mathrm{H}_{2} \mathrm{O}$ maser sources at $\mathrm{N}$ and $\mathrm{S}$ and a reference source, Cepheus $\mathrm{A}_{2} \mathrm{O}$ maser, simultaneously. The Cepheus $\mathrm{A}$ region is separated by 2 degrees from the NGC7538 region. The positions of $\mathrm{H}_{2} \mathrm{O}$ masers in $\mathrm{N}$ and $\mathrm{S}$ regions, distributed around the ultra-compact HII regions, are basically consistent with those found by means of interferometric observations of past 29 years. The masers may come from interface regions between the ultracompact HII regions and the environments of dense molecular gas.

8th European VLBI Network Symposium

September 26-29, 2006

Toruń, Poland

\footnotetext{
$1 \quad$ Speaker
} 


\section{Introduction}

NGC7538 region is a very active, massive star-forming area of the Perseus arm and the distance to it is estimated to $2.7 \mathrm{kpc}$ [e.g. 5]. According to single dish CO observations, there is a large molecular cloud complex, the size of which is 200 pc, and NGC7538 region seems to be at the northern edge of it. There is a high-density molecular cloud core toward the south of it [e.g. 5 and the references therein] (see Figure 1.). Three active star forming regions, IRS1-3, 9, and 11 exist in the cloud core [14]. These three infrared sources, being reflection nebulae, are associated with many other activities [11]. Sandel and others showed that each of the three regions is embedded within high-density dust and molecular cloud core [12]. CO high-velocity wing observations showed that the outflows are associated with the three regions [e.g. 6], and ultra-compact HII regions are also associated with each of the three regions [e.g. 8].
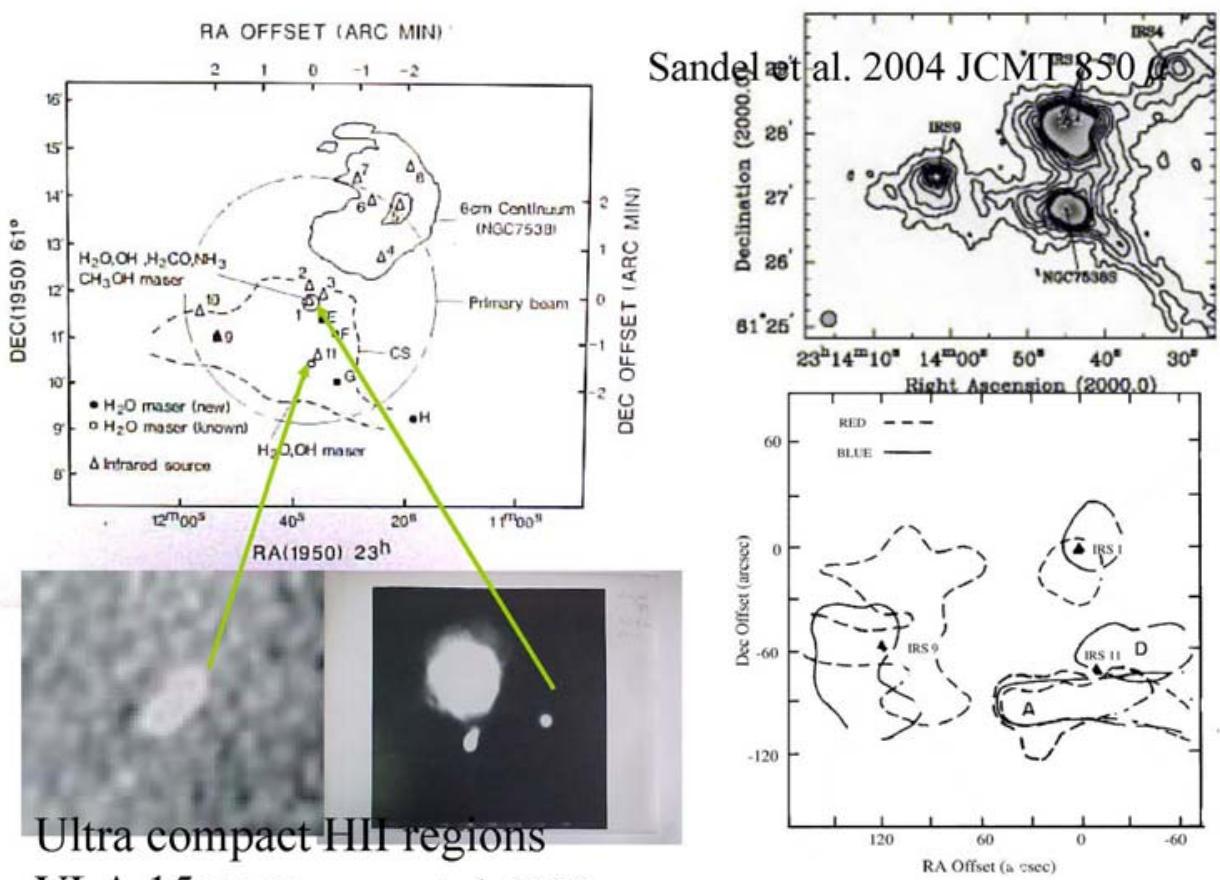

VLA $15 \mathrm{~cm}$ Kameya et al. 1990

Kameya et al. $1986 \mathrm{CO}$ outflow

Figure 1: Activity in NGC7538 region. Upper left panel: Infrared sources (triangles), masers, and HII region (NGC7538) [7]. The shape of CS molecular dense core is shown with dashed line[5]. Upper right panel: Dust distribution [12]. Bottom left panel: Ultra-compact HII regions at IRS1-3, and 11 [8]. Bottom right panel: distribution of the CO outflows at IRS1, 9, and 11 (triangles) [6].

Water masers, $\mathrm{OH}$ masers, and other masers are also associated with each of the regions. Especially ammonia, and formaldehyde masers are associated with IRS1 (or NGC7538 N) [1, 2, 4, 7, 8]. Recently, methanol masers have been discovered in IRS1-3, IRS9, and 11 [10]. Therefore, the common existence of star-forming activities suggests that these three regions are in similar phases of star formation. 
In 1987, water maser observation was carried out using Nobeyama Millimeter Array and many new maser sources were found [7]. What is most interesting is an elongated shape of the distribution of $\mathrm{H}_{2} \mathrm{O}$ maser spots at IRS11 (or NGC7538 S), which is the same as that of ultracompact HII region at IRS11, both in size and direction, and the direction of the CO outflow around IRS11 [6] (see bottom left panel of figure 2). The reflection nebula of IRS11 itself is situated northwest of the position of the ultra-compact HII region/water and $\mathrm{OH}$ masers, and it is in the blue lobe of molecular outflow. These distributions of the outflow of IRS11 masers suggest that CO outflow takes place at the position of the ultra-compact HII region/water and $\mathrm{OH}$ masers, and IRS11 reflection nebula is at the front side in the blue lobe. Hence, IRS11 region can be regarded as a typical area where star formation takes place.

What we want to know is the motion of water masers. To this end, the VLBI observations are required. Genzel and co-workers made the first VLBI observation of a water maser 29 years ago [1]. They proposed a model, in which masers appear at the expanding dense region around an ultra-compact HII region. Basically, their argument is correct and consistent with our model for IRS11, but their model is too simple to explain all the data because of lack of the information on the motion of the masers. Thus, new VLBI observations are needed in order to reveal the dynamics of this region.

\section{Observations}

We made two epochs of VLBI observations using VERA (VLBI Experiment of Radio Astrometry) consisting of four stations on Japanese islands. It was constructed five years ago and test VLBI observations have been carried out for several years. The baseline lengths range between 1000 and $2300 \mathrm{~km}$. The accuracy of the baseline lengths is of a few mm. VERA can observe at $22 \mathrm{GHz}$ and $43 \mathrm{GHz}$ bands of $\mathrm{H}_{2} \mathrm{O}$ masers and $\mathrm{SiO}$ masers, respectively [e.g. 3 and references therein]. It has the dual beam system, so it can cancel out the fluctuations of the atmosphere by simultaneous VLBI observations of masers and reference radio sources. We hope to measure parallax of masers with an accuracy better than $10 \%$ in the whole area of our galaxy in near future.

The 1st epoch observation was made on 13 Apr 2005. The recording system was DIR1000 (256 Mbps recording). The reference source was Cepheus $\mathrm{A}_{2} \mathrm{O}$ maser source, separated from the NGC7538 region by 2 degrees. Cepheus A is also a very active star-forming region [e.g. 13]. However, in this paper, we focus on the results of $\mathrm{H}_{2} \mathrm{O}$ distribution of NGC7538. These regions are at high declination, so the $u-v$ coverage was very good.

The second epoch observation was made on 27 Jul 2006. Details of that observation are the same except for the data recording system - DIR2000 (1 Gbps recording).

We used AIPS for the data reduction.

\section{Results and discussion}

\subsection{IRS11 (S) region}

We have detected all sources with a good signal-to-noise ratio at both epochs. The profile of $\mathrm{H}_{2} \mathrm{O}$ masers has changed during several tens of years but the maps of $\mathrm{H}_{2} \mathrm{O}$ maser spots show 
the similarity of their distribution. The maps of the maser spots at IRS11 are shown in Figure 2. There is an elongation towards northwest and southeast of maser spots positions. The results of the 2nd-epoch observation also show that similarity, even though the flux density of each spot has changed between the two epochs (470 days).

By comparing the positions of maser spots of the two epochs, we can see some positional displacement, however, we cannot measure maser motions using these data because of limitations imposed by its quality.

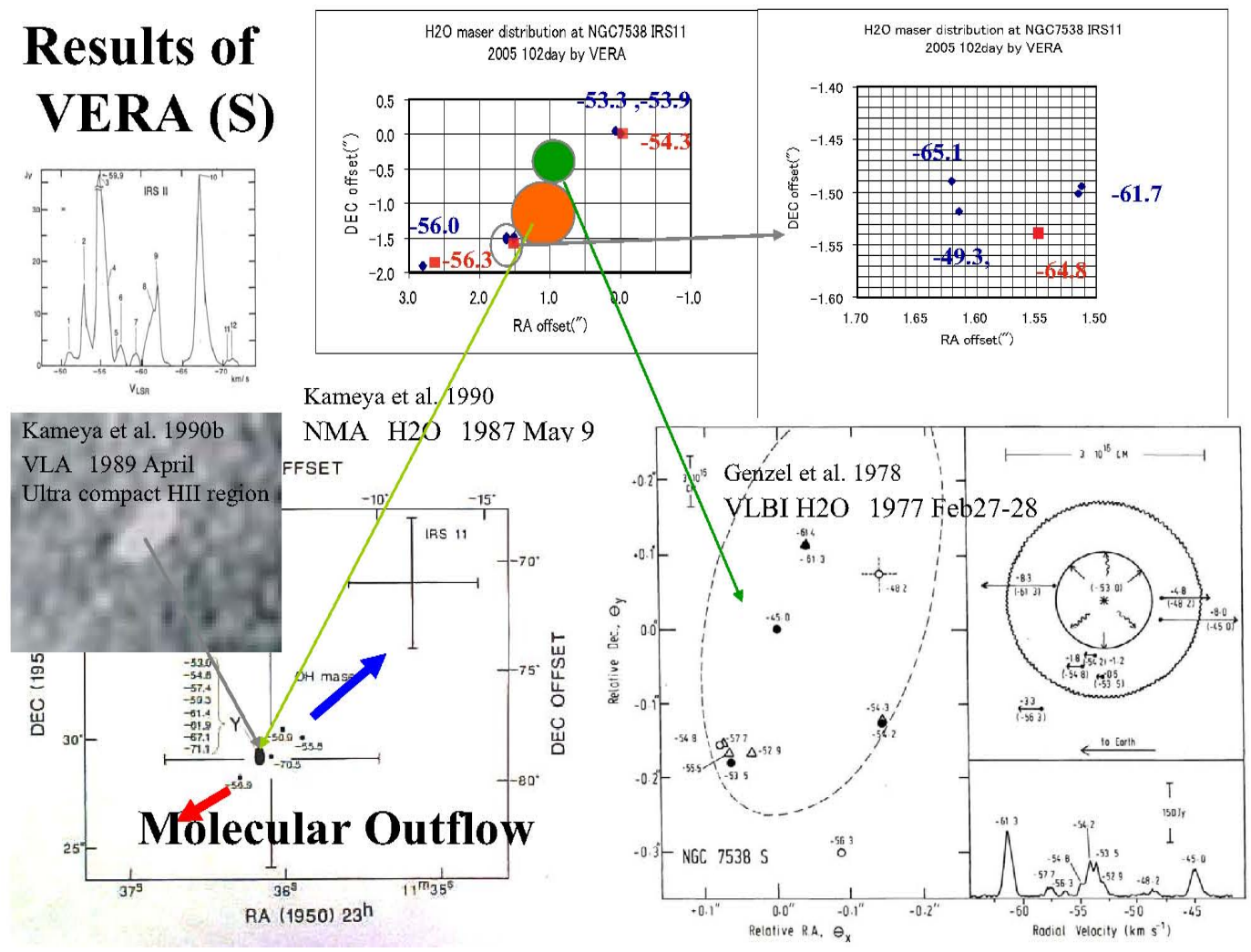

Figure 2: $\mathrm{H}_{2} \mathrm{O}$ maser distributions of NGC7538 IRS11 (S) region. Upper left panel: Spectrum of the $\mathrm{H}_{2} \mathrm{O}$ maser at NGC7538 IRS11 on 9 May 1987 [7]. Upper middle panel: The distribution of maser spots based on our observational results. Results of 1st epoch are shown by small triangle and diamond symbols; those of 2nd epoch are shown by rectangular symbols. Positions of masers in 1987 [7] and in 1977 [1] are shown with circles. The position of the offset centre $(0,0)$ is at the position of maser spots of Vlsr of $-53.9 \mathrm{~km} / \mathrm{s}$ at the $1 \mathrm{st}$ epoch and of $-54.3 \mathrm{~km} / \mathrm{s}$ at the $2 \mathrm{nd}$ epoch. Upper right panel: A close-up of maser spots as seen based on our observational results near RA offset of 1.6 arcsec and Dec offset of -1.5 arcsec. Bottom left panel: Distribution of masers at epoch of 1987 [7] together with an image of ultracompact HII region [8]. Positions of IRS11 and OH maser are shown with error bars, and the direction the CO outflow around IRS11 is shown with arrows. Bottom right panel: The VLBI results shown by Genzel et al. [1]. Details are described in the figure caption of Fig.12 of [1].

If the maser spot of the 2nd epoch is the same as that of the 1st epoch, the maser should have moved westerly with a velocity of more than several hundred $\mathrm{km} / \mathrm{s}$. This result is not consistent with the pattern of maser positions along outflow. Therefore, we think that the 
lifetime of maser spots may be less than 500 days. To verify that, we have begun more frequent maser VLBI observations.

Figure 1 also shows comparison of absolute maser positions between epochs in 1977, 1987, 2005, and 2006. There seems to be a displacement of maser spots, however, that displacement may be not real given their positional accuracy.

\subsection{IRS11 (N) region}

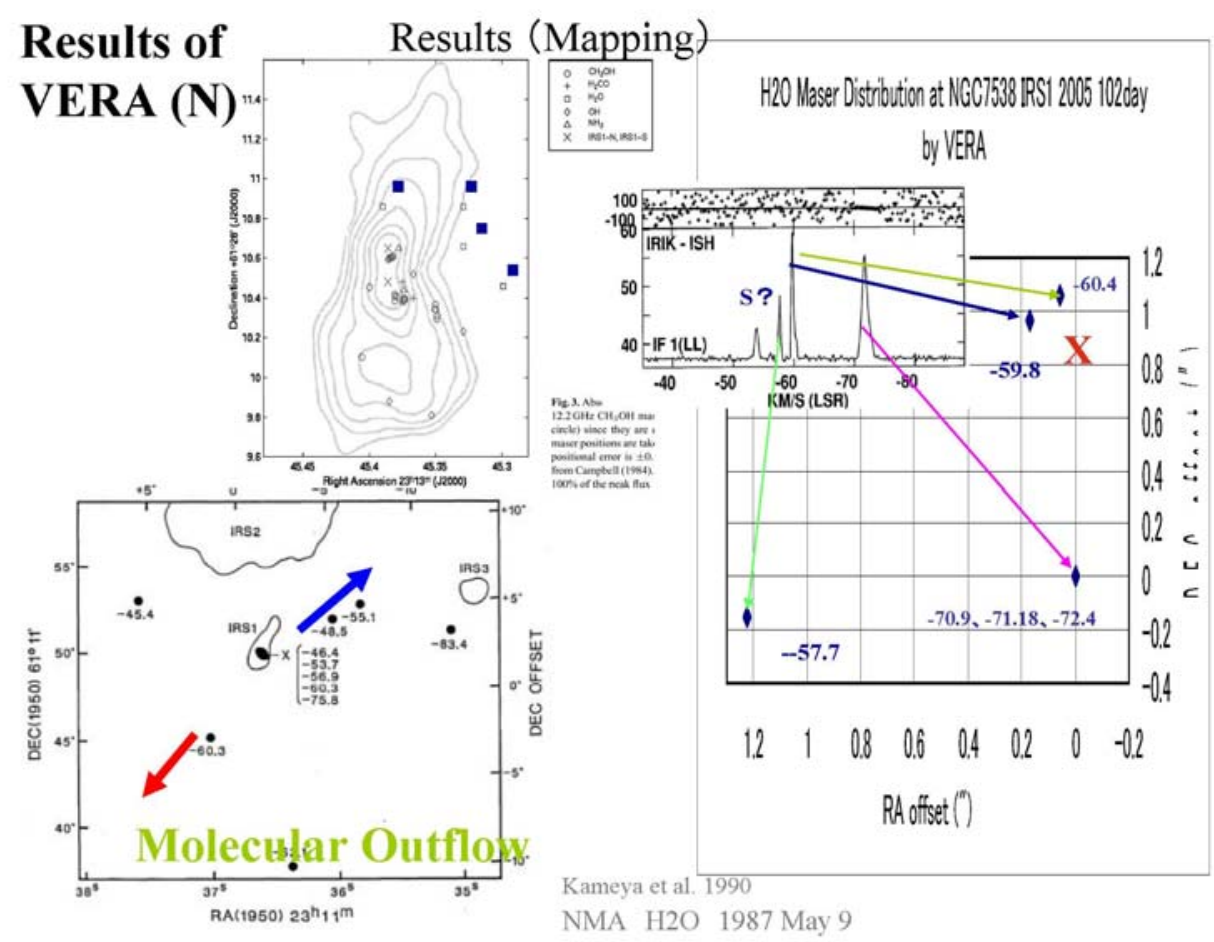

Figure 3: $\mathrm{H}_{2} \mathrm{O}$ maser distributions of NGC7538 IRS1 (N) region. Upper left panel: Absolute positions of different masers in NGC7538 IRS1 as shown in Figure 3 of Minier et al. [10]. (Details are given in the figure caption of the Figure 3 of [10].) The positions of water masers are shown with the filled rectangular symbols. Bottom left panel: Distribution of masers at epoch of 1987 [7] together with an image of ultra-compact HII regions at IRS 1, 2 and 3. Directions of the CO outflow around IRS1 are shown with arrows. Right panel: The distribution of maser spots based on our observational results. Results of the 1st epoch are shown with small diamond symbols. The position of the offset centre $(0,0)$ is at the position of maser spots of Vlsr of $-71.18 \mathrm{~km} / \mathrm{s}$. A cross-power spectrum of this region is also shown.

Figure 3 shows the results of our $\mathrm{H}_{2} \mathrm{O}$ masers for IRS1 region at the 1st epoch, and a detailed map of the distribution of maser spots, obtained for the first time. Basically, the distribution does not differ from the former results. Multi-epoch maps will also show motion of maser spots and make clear the dynamics of this region, and hopefully provide a parallax for this region. 


\section{Summary}

We made VLBI observations towards NGC7538 region using VERA at two epochs. The most detailed VLBI maps of $\mathrm{H}_{2} \mathrm{O}$ masers were obtained towards IRS1 and IRS11. The masers have survived for about 29 years and their distribution is basically the same and seems to appear around the ultra-compact HII regions.

\section{References}

[1] R. Genzel, D. Downes, J. M. Moran, K. J. Johnston, J. H. Spencer, R. C. Walker, A. Hashick, L. I. Matveyenko, L. R. Kogan, V. I. Kostenko, B. Ronnang, O. E. H. Rydbeck \& I. G. Moiseev, Structure and kinematics of $\mathrm{H} 2 \mathrm{O}$ sources in clusters of newly-formed OB stars, A\&Ap 66, 13 (1978)

[2] I. M. Hoffman, W.M. Goss \& P. Palmer, VLB A+Y27 images of the formaldehyde masers in $N G C 7538$ and $G 29.96$, Future Directions in High Resolution Astronomy: The $10^{\text {th }}$ Anniversary of the VLBA ASP Conference Series, Vol 340, ed, J. Romney \& M. Reid, 347 (2005)

[3] M. Honma, T. Fujii, T. Hirota, (and 25 other co-authors), First fringe detection with VERA's dualbeam system and its phase-referencing capability, PASJ, 55, L57 (2003)

[4] B. Hutawarakorn \& R. J. Cohen, OH masers, molecular outflows and magnetic field in NGC 7538, MNRAS, 345, 175 (2003)

[5] O. Kameya, T. I. Hasegawa, N. Hirano, M. Tosa, Y. Taniguchi, K. Takakubo \& M. Seki, CS and C34S observations of the NGC 7538 molecular cloud, PASJ, 38, 793 (1986)

[6] O. Kameya, T. I. Hasegawa, N. Hirano, K. Takakubo \& M. Seki, High-velocity flows in the NGC 7538 molecular cloud, ApJ, 339, 222 (1989)

[7] O. Kameya, K.-I. Morita, R. Kawabe \& M. Ishiguro, New $\mathrm{H}_{2} \mathrm{O}$ masers in the NGC 7538 region, ApJ, 355, 562 (1990)

[8] O. Kameya, N. Hirano, R. Kawabe \& B. Campbell, Detailed structure of the NGC7538 molecular cloud, in Proceedings of a Conference at the University of Manchester, March 26-30, Cambridge, ed, R. James, \& T. Millar (Cambridge University Press), 111 (1990b)

[9] S. Kraus, Y. Balega, M. Elitzur, K.-H. Hofmann, Th. Preibisch, A. Rosen, D. Schertl, G. Weielt \& E. T. Young, Outflows from the high-mass protostars NGC 7538 IRS1/2 observed with bispectrum speckle interferometry- Signature of flow precession, A\&Ap, 455, 521 (2006)

[10] V. Minier, R. S. Booth \& J. E. Conway, Observations of methanol masers in NGC7538: probable detection of a circumstellar disc, A\&Ap, 336, L5 (1998)

[11] D. K. Ojha, M. Tamura, Y. Nakamura, M. Fukagawa, K. Sugitani, C. Nagashima, T. Nagayama, T. Nagata, S. Sato, S. Vig, S. K. Ghosh, A. J. Pickles, M. Momose \& K. Ogura, A near-infrared study of the NGC 7538 star-forming region, ApJ, 616, 1042 (2004)

[12] G. Sandel \& A. Sievers, Submillimeter continuum observations of NGC7538, ApJ, 600, 269 (2004)

[13] J. M. Torrelles, M. A. Patel, J. F. Gomez, P. T. P. Ho, L. F. Rodriguez, G. Anglada, G. Garay, L. Greenhill, S. Curiel \& J. Canto, Discovery of linear "building blocks" of water masers shaping linear/arcuate microstructures in Cepheus A, ApJ, 560, 853 (2001)

[14] M. W. Werner, E. E. Becklin, I. Gatley, K. Matthews, G. Neugebauer \& C. G. Wynn-Williams, $A n$ infrared study of the NGC 7538 region, MNRAS, 188, 463 (1979) 